\section{Preparation, characterization and scale-up of sesamol loaded solid lipid nanoparticles}

\author{
Vandita Kakkar, Indu Pal Kaur \\ Department of Pharmaceutics, University \\ Institute of Pharmaceutical Sciences, \\ Panjab University, Chandigarh, India
}

\begin{abstract}
Sesamol loaded solid lipid nanoparticles (SSLNs) were prepared with the aim of minimizing its distribution to tissues and achieving its targeting to the brain. Three scale-up batches $(100 \times 1 \mathrm{~L})$ of S-SLNs were prepared using a microemulsification technique and all parameters were statistically compared with the small batch $(1 \times ; 10 \mathrm{~mL})$. S-SLNs with a particle size of less than $106 \mathrm{~nm}$ with a spherical shape (transmission electron microscopy) were successfully prepared with a total drug content and entrapment efficiency of $94.26 \pm 2.71 \%$ and $72.57 \pm 5.20 \%$, respectively. Differential scanning calorimetry and infrared spectroscopy confirmed the formation of lipidic nanoparticles while powder X-ray diffraction revealed their amorphous profile. S-SLNs were found to be stable for three months at $5 \pm 3^{\circ} \mathrm{C}$ in accordance with International Conference on Harmonisation guidelines. The SLN preparation process was successfully scaled-up to a $100 x$ batch on a laboratory scale. The procedure was easy to perform and allowed reproducible SLN dispersions to be obtained.
\end{abstract}

\section{Introduction}

Sesamol is the main antioxidant present in sesame oil. It is generated from sesamolin by roasting sesame seed or bleaching sesame oil. The antioxidant potential of sesamol has been established in vitro and its antimutagenic, chemoprotective, antiaging, cardioprotective and hepatoprotective effects are also well documented. ${ }^{1-5}$ The physicochemical nature of sesamol (log P 1.29; solubility $38.8 \mathrm{mg} / \mathrm{mL}$ ) substantially enhances its distribution to the tissues, thus minimizing its delivery to the brain. This led us to look for an alternative controlled release drug carrier system ${ }^{6}$ such that the brain could be efficiently targeted.

Solid lipid nanoparticles (SLNs) consist of spherical solid lipid particles in the nanometer range dispersed in a solution of surfactant which facilitates their passage across the biological membrane. ${ }^{7}$ Although there are numerous methods for the preparation of SLNs, only a few of them can be easily scaled-up.

The high pressure homogenization technique, which has been used since the 1950 s for the production of parenteral emulsions, is the most common technique, and is used to produce large volumes of SLN dispersions. However, this manufacturing process requires large energy resources. Furthermore, the process is not suitable for the production of water soluble drugs that may partition towards the water phase as a result of the homogenization pressure, resulting in low entrapment efficiencies. ${ }^{8}$

Still another efficient and robust method of producing SLNs is by microemulsification. This involves SLNs being prepared by the dispersion of warm oil-in-water (o/w) microemulsion in cold water $\left(0-4^{\circ} \mathrm{C}\right)$; solid lipids with high melting points forming the internal phase of the microemulsions. ${ }^{9}$ Although scaling-up of this method for parenterals was reported by Marengo et al. in 2000, they scaled-up the process for $1 \mathrm{~mL}$ of microemulsion to only 100 $\mathrm{mL}$ using specially designed apparatus. The warm liquid microemulsion was sterilized through a $0.22 \mathrm{~mm}$ membrane filter; the nanodroplet size of warm o/w microemulsion is lower than $100 \mathrm{~nm}$. After passing through the filter, the warm microemulsion was made to flow through the interchangeable needle and drop directly into a stirred cold aqueous medium. ${ }^{10}$ Small batches of $5 \mathrm{~mL}$ microemulsion poured into $50 \mathrm{~mL}$ of cold water (1:10 dilution) were being produced in our laboratory before this. The pharmacodynamic study of the prepared SLNs in an ovariectomized rat model proved our intention to deliver significant amount of sesamol to brain. ${ }^{11}$

However, in order to test the commercial viability of the process, we planned to develop the scaled-up batches of 1 liter (L) or more and characterize the SLN formulation that develops according to various in vitro parameters, including particle size, percentage entrapment efficiency (\%EE), transmission electron microscopy (TEM), infrared spectroscopy (IR), differential scanning calorimetry (DSC), powder X-ray diffraction studies (PXRD) and in vitro release. We aimed to produce three scaled-up batches (1 L each) of sesamol SLNs using a microemulsification method and appropriately characterized them. Further objective of the study was to obtain a concentrated dispersion of nanoparticles by effecting only a 1:1 dilution of the hot microemulsion with cold water instead of a commonly 1:10 or a 1:50 dilution. Production of a highly diluted SLN dispersion is one of the major limitations of the microemulsification method. The method requires subsequent steps such as diafiltration or lyophilization in order to obtain a stable concentrated product, followed by reconstitution for compliant usage. Both lyophilization and reconstitution can result in
Correspondence: Indu Pal Kaur, University Institute of Pharmaceutical Sciences, Panjab University, Chandigarh-160014, India.

Tel. +91.172.2534191 - Tele Fax: 91.172.2543101.

E-mail: indupalkaur@yahoo.com

Key words: sesamol, solid lipid nanoparticles, microemulsification, scale-up.

Received for publication: 17 October 2011. Revision received: 21 February 2011. Accepted for publication: 4 April 2012.

This work is licensed under a Creative Commons Attribution NonCommercial 3.0 License (CC BYNC 3.0).

(C) Copyright V. Kakkar and I.P. Kaur, 2012 Licensee PAGEPress, Italy

Nanotechnology Development 2012; 2:e8 doi:10.4081/nd.2012.e8

aggregation of particles to a micron size. So the other very important consideration of the study was to try and develop a one-step, concentrated and ready to use dosage form without any further treatment.

\section{Materials and Methods}

\section{Materials}

Sesamol was obtained from Jubilant Life Sciences (Noida, Uttar Pradesh, India); soy lecithin (Hi Media, Mumbai, India), Tween 80 (S.D. Fine Chemicals Ltd., Mumbai, India), and Compritol $^{\circledR} 888$ ATO (glyceryl behenate, a gift sample from Gattefosse, Paramus, NJ, USA) were also used in the study. All other chemicals and reagents were of analytical grade and were used without further purification.

\section{Preparation of scaled-up batches of sesamol loaded solid lipid \\ nanoparticles}

Small batches $(1 \times)$ of sesamol loaded solid lipid nanoparticles (S-SLNs) were produced using $10 \mathrm{~mL}$ glass beakers to maintain the lipidic and aqueous phases at temperature of $80-85^{\circ} \mathrm{C}$. Suitable quantities were taken to produce a $5 \mathrm{~mL}$ of microemulsion which was poured into equal volume of ice-cold $\left(0-4^{\circ} \mathrm{C}\right)$ water under magnetic stirring for the formation of solid lipid nanoparticulate dispersions. For production of a 100x batch of S-SLNs, 500 $\mathrm{mL}$ of microemulsion was produced. This was poured into an equivalent amount of ice-cold water $(500 \mathrm{~mL})$ to provide a concentrated dispersion. All the ingredients were weighed proportionally to produce a $100 \mathrm{x}$ batch. The process involved placing polysorbate 80 (250 $\mathrm{mL})$ soy lecithin $(80 \mathrm{~mL})$, and water $(180 \mathrm{~mL})$ 
together in a beaker which was heated to the lipid melt temperature. Glyceryl behenate (40 g) was melted separately at $82-85^{\circ} \mathrm{C}$. Sesamol (1:10 with respect to lipid) was added to the aqueous phase, following which the hot aqueous emulsifier mix was dropped at once into the lipid melt under magnetic stirring to obtain a clear microemulsion. The hot microemulsion thus formed was poured slowly in a streamline into an equivalent amount of cold water $\left(\sim 2^{\circ} \mathrm{C}\right)$ under continuous mechanical stirring (5000 rpm) for exactly $2 \mathrm{~h}$. SLNs are formed in the aqueous medium by crystallization of high melting point lipid droplets that represent the oil phase of the microemulsion. ${ }^{12}$ The prepared SLNs were stored in a refrigerator until further analysis. Three scaled-up batches were produced to check reproducibility of the selected method. All three were characterized using suitable parameters to ensure complete in vitro characterization and the results were also compared for uniformity of the parameters with the $1 \mathrm{x}$ laboratory scale batch.

\section{Characterization of sesamol loaded solid lipid nanoparticles}

\section{Particle size analysis}

The mean diameter of SLNs in the dispersion (with appropriate dilutions with triple distilled water) was determined using photon correlation spectroscopy (Zeta sizer 2000, Malvern Instruments Ltd., Malvern, UK).

\section{Transmission electron microscopy}

SLN morphology was examined using an electronic transmission microscope (Hitachi H-100; Hitachi Kokusai Electric Inc., Tokyo, Japan).

\section{Differential scanning calorimetry}

DSC was performed with a Perkin-Elmer differential calorimeter. DSC is a tool to investigate the melting and crystalline behavior of materials like SLNs. ${ }^{13,14}$ The breakdown or fusion of the crystal lattice by heating or cooling the sample provides information about the internal polymorphism, crystal ordering or glass transition processes. ${ }^{15}$ It uses the fact that different lipid modifications possess different melting points and enthalpies. The thermal analysis of the pure drug, lipid and SLNs were carried out to look for any significant changes in the pattern of the peaks. Samples were placed in a conventional aluminum pan and heated from $10^{\circ} \mathrm{C}$ to $250^{\circ} \mathrm{C}$ at a scan speed of $10^{\circ} \mathrm{C} / \mathrm{min}$.

\section{Infrared spectroscopy}

IR spectroscopy is used to elaborate the structure and stereochemistry of the bulk material and the nanoparticles. ${ }^{16}$ The drug, the lipid, and the formulated SLNs were analyzed.
The peaks obtained for the free drug and lipid, and their physical mixture was compared with the lyophilized SLN formulation to look for any significant changes.

\section{Powder X-ray diffraction}

The crystalline/amorphous nature of formulated nanoparticles was confirmed by X-ray diffraction measurements carried out with an Xray diffractometer (XPERT-PRO, PANalytical, Almelo, The Netherlands). PXRD studies were performed by exposing the samples to $\mathrm{CuK}_{\alpha}$ radiation $(45 \mathrm{kV}, 40 \mathrm{~mA})$ and scanning from $5^{\circ}$ to $50^{\circ}, 2 \theta$ at a step size of $0.017^{\circ}$ and scan step time of $25 \mathrm{~s}$. Samples used for PXRD analysis were the same as those used for DSC. The instrument measures interlayer spacing $d$ which is calculated from the scattering angle $\theta$, using Bragg's equation $\mathrm{n} \lambda=2 \mathrm{~d} \sin \theta$ where $\lambda$ is the wavelength of the incident $\mathrm{X}$-ray beam and $n$ is the order of the interference. Obtained XRD patterns were compared for characteristic drug peak intensity.

\section{Total drug content}

Total drug content (TDC) was estimated by spectrophotometry at $\lambda_{\text {max }}$ of $294 \mathrm{~nm}$ by disrupting $1 \mathrm{~mL}$ of the SLN dispersion using an appropriate volume of chloroform: methanol (1:1).

\section{Entrapment efficiency}

A dialysis bag with a cut off of $12 \mathrm{KDa}(\mathrm{Hi}$ Media) was used to estimate the EE. We poured $1 \mathrm{~mL}$ of S-SLN dispersion $(3.72 \mathrm{mg} / \mathrm{mL}$ ) in the dialysis bag, both ends of which were tied tight to prevent any leakage. The bag was dipped in $100 \mathrm{~mL}$ of water stirred magnetically at $150 \mathrm{rpm}$. Dialysate was withdrawn after 15 min and analyzed by spectrophotometry; time was optimized using an equivalent amount of free sesamol to be released in water; this was performed assuming no amount of sesamol is entrapped within the SLNs.

$$
\begin{gathered}
\begin{array}{c}
\% \mathrm{EE}=\text { Total drug } \\
\text { entrapped/Total drug }
\end{array} \\
\text { content x } 100
\end{gathered}
$$

Absorbance value obtained for blank SLNs treated in a similar manner was used as the control value to compensate for any interference with the ingredients. All measurements were taken in triplicate for all the three batches produced. Amount of drug in the dispersion retained inside the dialysis bag gave a direct measure of the quantity of drug entrapped.

Values obtained for the amount of drug in the supernatant and that retained in the dispersion inside the dialysis bag were added together to confirm the mass balance.

\section{In vitro drug release}

The in vitro release studies were carried out by the dialysis membrane method for studying the release of sesamol from the developed SLNs. ${ }^{15}$ The receptor media used for the studies was made up of $100 \mathrm{~mL}$ of phosphate buffer, $\mathrm{pH} 7.4$, pre-equilibrated at $37 \pm 0.5^{\circ} \mathrm{C}$. Aqueous SLN dispersion (1 mL containing $3.72 \mathrm{mg}$ of sesamol as per the drug content studies) was placed in the dialysis tubing which was then sealed at both ends and dipped into the receptor media, maintained at $37 \pm 0.5^{\circ} \mathrm{C}$ and stirred continuously at $150 \mathrm{rpm}$. Samples of $3 \mathrm{~mL}$ each were withdrawn from the receptor medium with replacement at various time intervals and analyzed by spectrophotometry at $294 \mathrm{~nm}$. All measurements were taken in triplicate for the three scale-up batches and also for the $1 x$ labscale batch. Results obtained were analyzed statistically to confirm the reproducibility of the large scale batches produced using the microemulsification technique.

\section{Stability}

S-SLNs were stored in vials at $5 \pm 3^{\circ} \mathrm{C}$ for three months and the samples were withdrawn at 0,1 and 3 months, as per International Conference on Harmonisation (ICH) guidelines. The average size, total drug content and the entrapment efficiency were determined at each time point.

\section{Results}

\section{Characterization of sesamol loaded solid lipid nanoparticles}

\section{Particle size}

Average particle size of all the three $100 \mathrm{x}$ batches was found to be $106.6 \mathrm{~nm}$ or under (Figure 1) with the actual size being 95.35, 98.11 and $106.6 \mathrm{~nm}$ for batches I, II and III. PI were $0.432,0.482$ and 0.303 for the three batches, respectively. Particle size and the PI obtained for the 1x batch were $122 \mathrm{~nm}$ and 0.255 , respectively.

\section{Transmission electron microscopy}

SLNs were found to be spherical in shape by TEM (Figure 2). The size of the lipidic nanoparticles observed under TEM (40-90 nm) was close (though smaller) to the results obtained using photon correlation spectroscopy.

\section{Differential scanning calorimetry}

DSC is a thermoanalytical technique in which the difference in the amount of heat required to maintain the sample and reference at same temperature is measured as a function of temperature and time. The basic principle underlying this technique is that when the sample undergoes a physical transformation 
(such as melting, desolvation), some amount of heat is required to flow through it, depending on whether the process is exothermic or endothermic, to maintain both reference and sample at the same temperature. DSC measures this heat flow into or from the sample when it is heated or cooled. These measurements provide qualitative and quantitative information about physicochemical changes (i.e. endothermic, exothermic processes or changes in heat capacity). Figure 3 shows DSC thermograms of pure sesamol, Compritol ${ }^{\circledR} 888$ ATO and the formed SLNs. The DSC curve for pure sesamol showed a fusion endotherm at $65.43^{\circ} \mathrm{C}$ corresponding to the melting point of sesamol $\left(60-65^{\circ} \mathrm{C}\right)$ while the physical mixture shows peaks at $71.13^{\circ} \mathrm{C}$ (corresponding to the lipid) and another peak at $162.28^{\circ} \mathrm{C}$ (corresponding to the degradation peak of sesamol). Pure Compritol ${ }^{\circledR} 888$ AT0 shows an endothermic peak at $73.06^{\circ} \mathrm{C}$. It also showed a degradation peak at over $160^{\circ} \mathrm{C}$. However, DSC of developed SLNs did not show a peak corresponding to sesamol, confirming the successful incorporation of the drug into the SLNs. A broad endotherm starting from $95.17^{\circ} \mathrm{C}$ and showing two distinct peaks at $99.97^{\circ} \mathrm{C}$ and $112.36^{\circ} \mathrm{C}$ was observed for the SLNs, although a small shoulder was observed near the lipid melting point of $73.06^{\circ} \mathrm{C}$. Observation of broad peaks indicates the amorphous nature of the developed SLNs. DSC for the laboratory scale (1x) batch and the $100 x$ batch were similar.

\section{Infrared spectroscopy}

The IR peaks obtained with the developed formulation of S-SLNs revealed an intermolecular stretching of the -OH group (3400-3200 $\mathrm{cm}^{-1}$ ) of the drug when compared with the peak of pure sesamol (Figure 4). This is a direct indication of the formation of SLNs as the stretching could not be observed when the physical mixture of the same components were analyzed (Figure 4). Thus, the formation of SLNs could be confirmed by IR analysis.

\section{Powder X-ray diffraction studies}

Overlaid PXRD patterns of sesamol, Compritol $^{\circledR} 888$ AT0, lyophilized blank SLN (BSLN) and S-SLN are shown in Figure 5. The PXRD pattern of sesamol showed sharp peaks at $2 \theta$ scattered angles 7.12, 15.19, 18.08 and 18.360 which indicated its crystalline nature. However, no characteristic peaks for sesamol in lyophilized S-SLNs were observed, indicating the amorphous nature of the formed SLNs. PXRD pattern of Compritol ${ }^{\circledR} 888$ ATO shows sharp peaks at $2 \theta$ scattered angles 21.16, 23.37, 23.52 and $35.76 \theta$; indicating the crystalline state of Compritol ${ }^{\circledR} 888$ ATO.

Total drug content and entrapment efficiency

TDC and EE of S-SLNs (100x batch) was

\section{Sample Details}



General Notes:

File Name: carbon nano tubes of 21-... Dispersant Name: Water

Record Number: 37

Material Rt 1.59

Dispersant Rl: 1.330

Viscosity (cP): 0.8872

Material Absorbtion: 0.01

Measurement Date and Time: Thursday. April 21. $20112: 42$.

Results

$\begin{array}{rllll} & & \text { Diam (nm) } & \text { \% Intensity } & \text { Width (nm) } \\ \text { Z-Average (d.nm): } 100.6 & \text { Peak 1: } & 164.3 & 96.9 & 99.44 \\ \text { Pdil: } 0.303 & \text { Peak 2: } & 12.85 & 3.1 & 2.455 \\ \text { Intercept: } 0.952 & \text { Peak 3: } & 0.000 & 0.0 & 0.000\end{array}$

Result quality : Good

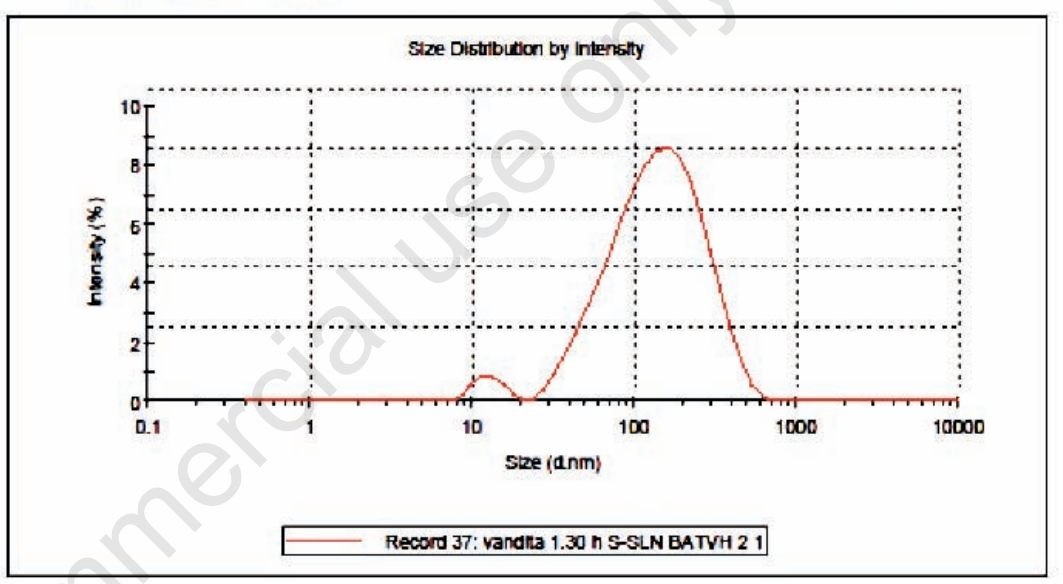

Figure 1. Particle size report (100x; batch III) as obtained after photon correlation spectroscopy analysis.

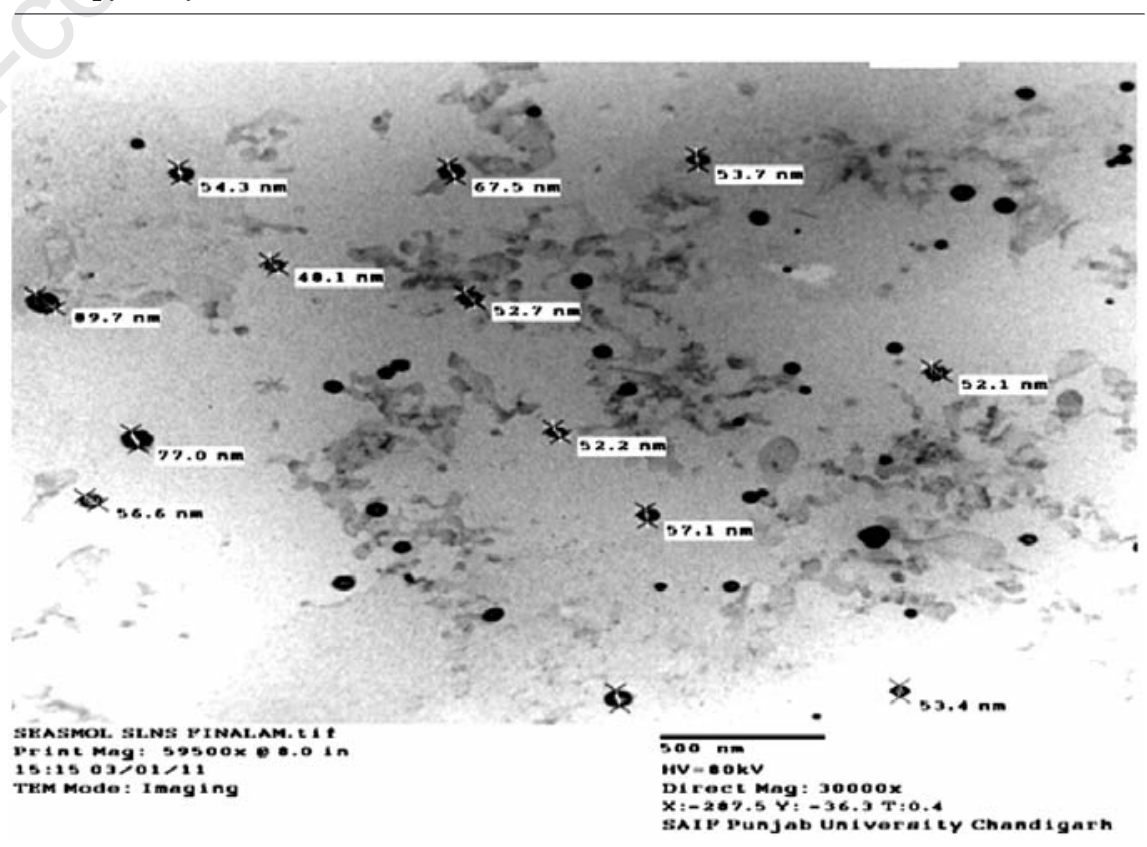

Figure 2. Transmission electron microscopy micrograph of sesamol loaded solid lipid nanoparticles. 
estimated to be $94.26 \pm 2.71 \%$ and $72.57 \pm 5.20 \%$ $(n=9)$, respectively, and there was no significant difference $(\mathrm{P} \leq 0.05)$ between the TDC and $\mathrm{EE}$ of the three batches produced and the small scale batch (1x) (TDC-91.28 $\pm 3.28 \%$; EE$67.28 \pm 3.86 \%)$. High (EE) values $(\sim 70 \%)$ indicate the efficiency of the method for preparation of sesamol loaded SLNs and over 90\% TDC confirms drug losses during formulation were insignificant. A significant EE also shows the suitability of the components and their relative proportion in the formulated SLNs.

\section{In vitro drug release}

The drug release from S-SLNs at $37 \pm 0.5^{\circ} \mathrm{C}$ is shown in Figure 6. The release of sesamol from S-SLNs was fitted to a first order kinetics model and occurs by diffusion. The release was prolonged for up to $24 \mathrm{~h}$ with approximately $50 \%$ of the drug being released before $4 \mathrm{~h}$ (Figure 6). Almost 90\% of the drug was released in less than $16 \mathrm{~h}$ which may be due to the water soluble nature of sesamol and hence its incorporation into the outer phospholipid layer of the lipidic nanoparticles. However, a prolonged release was observed in the later stage (up to $24 \mathrm{~h}$ ) which may be attributed to the diffusion of the remaining drug from the lipidic core. Overall, the system followed a nonFickian drug release. In case of the $1 \mathrm{x}$ batch, a slower release was obtained and a total release of $68 \%$ was achieved at the end of $24 \mathrm{~h}$.

\section{Stability}

After three months of storage at $5 \pm 3{ }^{\circ} \mathrm{C}$, the S-SLNs were found to be stable according to ICH guidelines without any significant increase $(\mathrm{P} \leq 0.05)$ in particle size (Table 1$)$. Change in EE (5.2\%) and TDC (2.2\%) at three months was also without significance $\left(\mathrm{P}_{\leq}\right.$ 0.05 ) with respect to the 0 time samples, indicating a stable formulation.

\section{Discussion}

The main aim of this research was to scale up the preparation process of SLNs so as to obtain a highly stable concentrated dispersion. The study also aimed to design a process which can be carried out very easily and using relatively unsophisticated equipment. The use of the simplest equipment possible would provide scope for its commercial launch even by those conventional companies who specialize in simple pharmaceutical products such as emulsions. The phytochemical industry is also ready to venture into the development of $p h y$ topharmaceutical products that involve a minimum investment in sophisticated equipment.

A stable microemulsion was formed even when it was prepared in a 100 -fold $(500 \mathrm{~mL})$

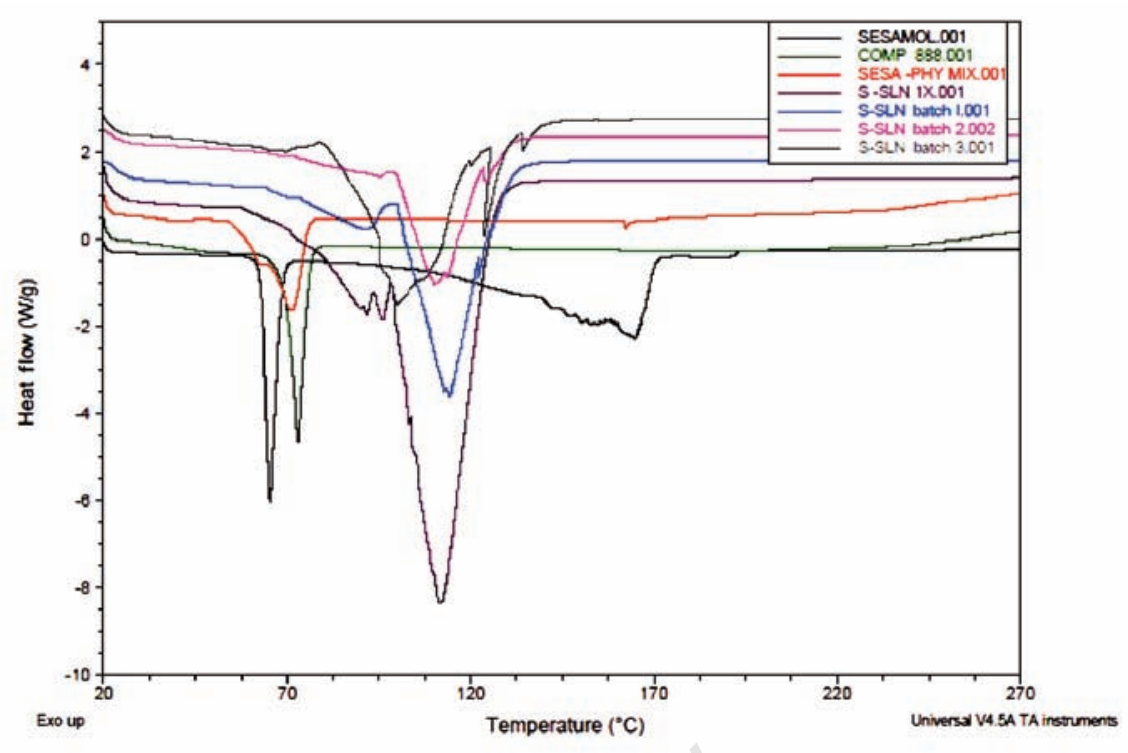

Figure 3. Differential scanning calorimetry thermograms of pure sesamol, Compritol ${ }^{\oplus}$ 888 ATO, their physical mixture and the formed solid lipid nanoparticles (SLNs) batches (1 $x$ and three 100x batches).

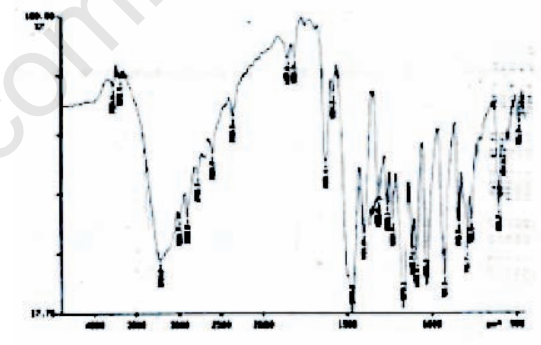

Sesamol

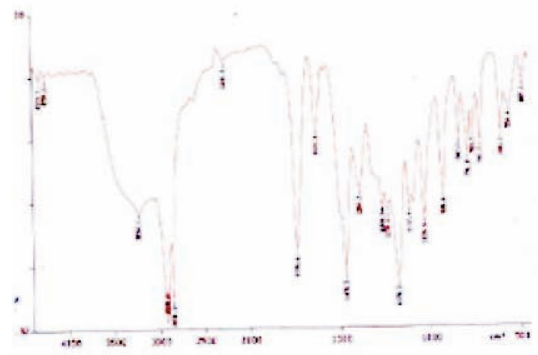

Physical Mixture
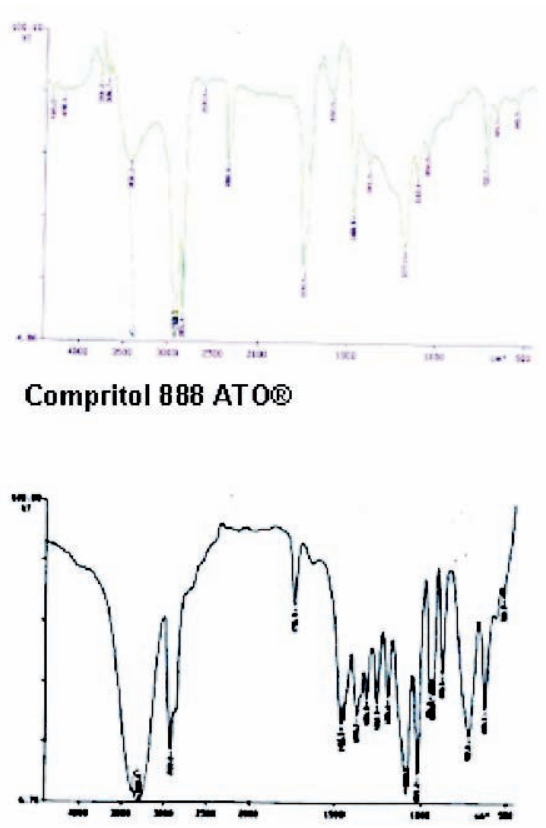

Compritol 888 ATOQ SLNs
Figure 4. Infrared spectroscopy analysis of sesamol, Compritol ${ }^{\circledR} 888$ ATO, physical mixture and $\mathrm{Compritol}^{\circledR} 888$ ATO solid lipid nanoparticles (SLNs). 
batch volume when compared to the small $1 x$ laboratory batch. The microemulsion formed needs to be stable for a sufficient period of time to allow it to be poured into cold water as slowly and smoothly as possible under mechanical stirring (5000 rpm). This process can now be completed in $30 \mathrm{~min}$, with $5-10 \mathrm{~min}$ of pouring time and $15 \mathrm{~min}$ of continued mechanical stirring, even after the complete addition of microemulsion. In the small scale (1x) batch, we were following stirring for long periods of time (3-4 h) after transfer of the microemulsion in order to achieve a small particle size.

In our previous experiments, o/w microemulsion was dispersed using a 18 gauge needle attached suitably to a glass syringe as reported and recommended (even for scaledup batches) by other workers. ${ }^{10,17}$ Even though the process always resulted in a small particle size $(<200 \mathrm{~nm})$, it is demanding and difficult to use a hot syringe as the emulsion constitutes a high melting lipid, so any cool surface results in congealing of the lipidic phase; neither is the process suitable to be scaled-up easily on a laboratory scale. To overcome the problem, we tried to pour the microemulsion directly into cold water. We could successfully produce SLNs with the required particle size and a low polydispersity index, and the process could be suitably scaled-up to obtain a concentrated (1:1 dilution) SLN dispersion (1 L), with an average particle size as small as $95 \mathrm{~nm}$ (PI of 0.3 ) for one of the batches. However, PI is not a very important issue for formulations which are administered orally. ${ }^{18}$

SLNs are formed by rapid quenching of the warm $0 / w$ microemulsion and the temperature difference between the warm microemulsion and the cold dispersing water plays an important role in determining the size of SLN. ${ }^{19} \mathrm{~A}$ rapid crystallization of the oil droplets of the warm microemulsion during quenching favors the formation of small SLNs, avoiding the coalescence among the oil nanodroplets.

The aqueous system made up of sesamol in polysorbate 80 and soy lecithin, while Compritol ${ }^{\circledR} 888$ AT0 was chosen as the lipid component as it results in dispersions with small particle size. ${ }^{7,20}$ The main reason for the selection of Compritol ${ }^{\circledR} 888$ AT0 as lipidic phase is its unique composition of glycerol tribehenate (28-32\%), glycerol dibehenate (52$54 \%$ ) and glycerol monobehenate (12-18\%). The most abundant fatty acid is behenic acid (>85\%) but other fatty acids (C16-C20) are also present. Compritol ${ }^{\circledR} 888$ ATO has an amphiphilic character due to the presence of partial acylglycerols. Its hydrophilic lipophilic balance (HLB) is approximately 2, having a melting point between $69^{\circ} \mathrm{C}$ and $74^{\circ} \mathrm{C}$ and a density value of $0.94 \mathrm{~g} / \mathrm{cm}^{3}$. Compritol ${ }^{\circledR} 888$ AT0 has a peroxide value lower than 6 meq $\mathrm{O}_{2} / \mathrm{kg}$ indicating high chemical stability. It is thought that a lipid containing a major portion of diglycerides would be better able to hold an amphiphilic drug and would thus result in more efficient entrapment. Sesamol shows a solubility of $38.8 \mathrm{mg} / \mathrm{mL}$ and a $\log \mathrm{P}$ of 1.29 , favoring the octanol phase, thus showing it to be an amphiphilic molecule. The results obtained with the scaled-up batches of S-SLNs were found to be reproducible. Also, there was no difference between the characterization parameters of the $1 \mathrm{x}$ and $100 \mathrm{x}$ batches. There was no significant difference $(\mathrm{P} \leq 0.05)$ between the particle size, drug content and entrapment efficiency either within the scaled-up batches or the $1 \mathrm{x}$ batch, indicative of the robustness of the method. However, a sig-

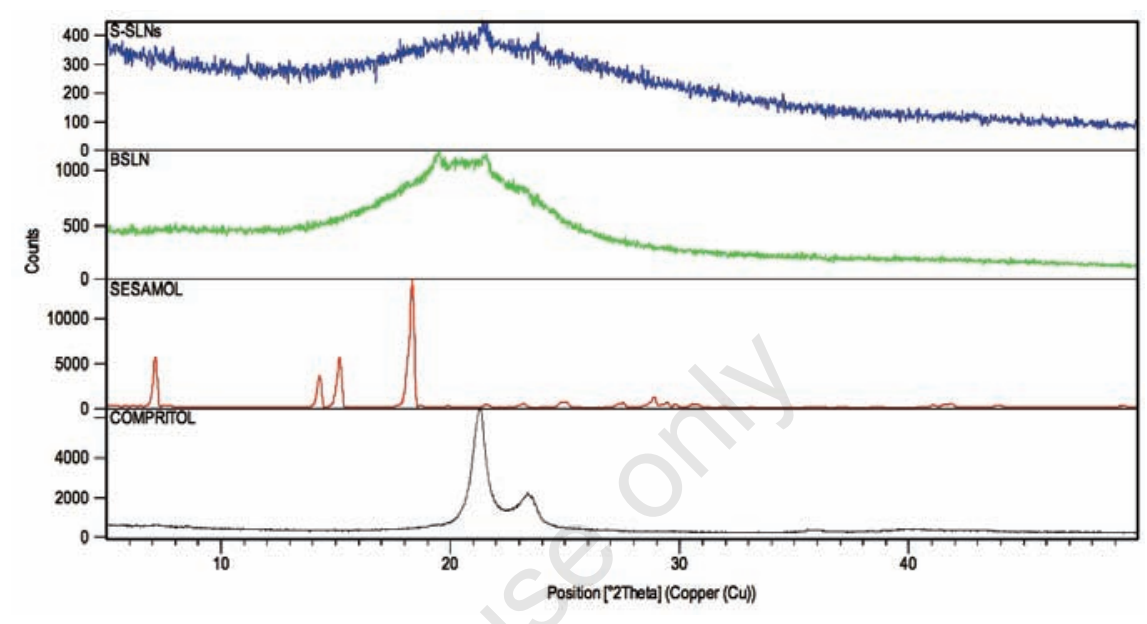

Figure 5. Overlaid powder X-ray diffraction studies patterns of Compritol $^{\circledR} 888$ ATO, $^{\circ}$ sesamol, blank solid lipid nanoparticles (BSLN) and sesamol loaded solid lipid nanoparticles (S-SLNs).

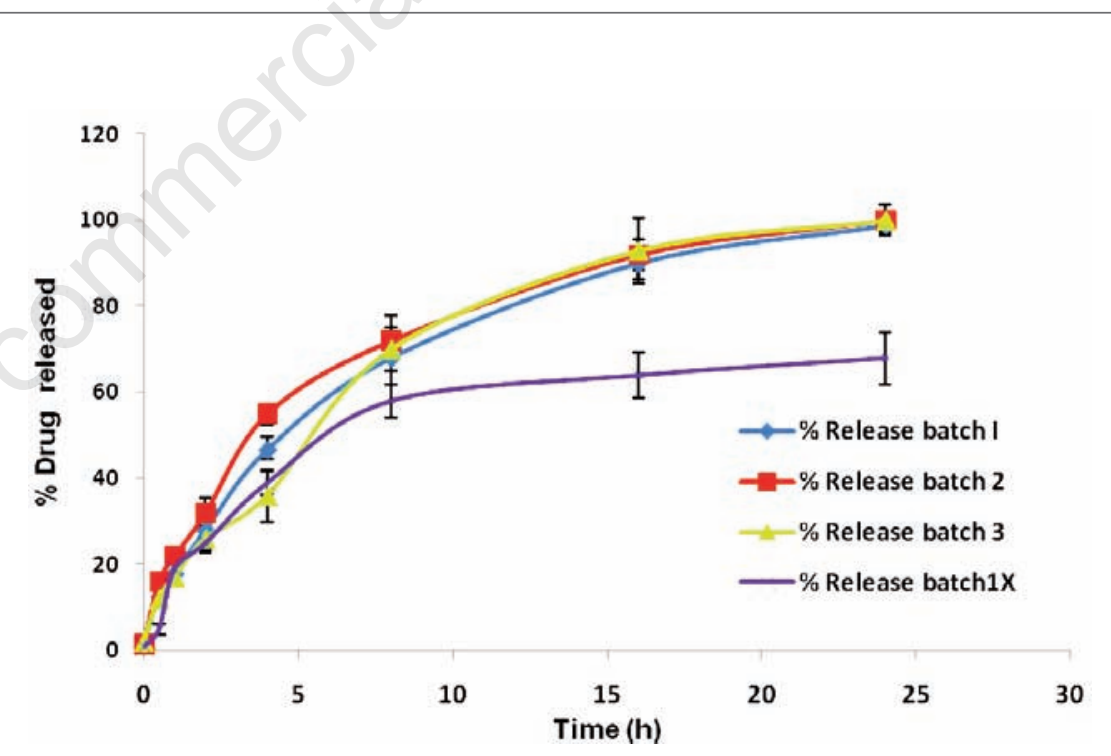

Figure 6. In vitro release profile of sesamol, from sesamol loaded solid lipid nanoparticles, by dialysis.

Table 1. Stability study parameters during storage of sesamol loaded solid lipid nanoparticles at $5 \pm 3^{\circ} \mathrm{C}$.

\begin{tabular}{lccc} 
Time points & $\begin{array}{c}\text { Av. particle sive } \\
(\mathrm{nm})\end{array}$ & $\begin{array}{c}\text { Total drug content } \\
(\%)\end{array}$ & $\begin{array}{c}\text { Entrapment efficiency } \\
(\%)\end{array}$ \\
0 time & $106 \pm 19.60$ & $94.26 \pm 2.71$ & $72.57 \pm 5.20$ \\
1 month & $95 \pm 22.00$ & $92.70 \pm 4.16$ & $71.60 \pm 1.49$ \\
\hline 3 month & $109 \pm 15.60$ & $92.30 \pm 1.58$ & $68.30 \pm 2.15$ \\
\hline
\end{tabular}


nificant difference $(P \leq 0.05)$ was observed for the cumulative amount released for the $1 \mathrm{x}$ batch which was only $68 \%$ up till $24 \mathrm{~h}$ and the $100 \mathrm{x}$ batch ( $>90 \%$ at $24 \mathrm{~h}$ ). This difference may be due to the amount of surfactant left unused after the formulation of SLNs. Scaledup batches may accumulate a higher percentage of surfactant both on the surface of the formed nanoparticles and/ or in the surrounding aqueous phase, helping the drug to diffuse out more rapidly, especially the drug entrapped in the inner lipidic core to the outer aqueous phase. The high reproducibility of results with respect to the characterization of SLNs may be due to an optimized formula for producing the microemulsion. Final composition of the microemulsion resulted in its spontaneous formation with an average particle size of less than $40 \mathrm{~nm}$. When poured in ice cold water under high speed stirring this was expected to result in solid particles in the nanometric range. Stability of the formulation for up to three months was in accordance with ICH guidelines. Several cases report that SLNs show an inadvertent increase in particle size when kept and stored under refrigerated conditions, due to the gelling of the lipid phase. We, however, did not observe any such gelling phenomenon, which may be due to the high viscosity imparted by the concentrated SLN dispersion. A high concentration of polysorabte $80>20 \%$ may also contribute to the high viscosity which may also help the nanoparticles to remain in a dispersed form without any aggregate formation.

Also, in other methods used for scaling-up solid lipid nanoparticles, especially high pressure homogenization, in general, a large number of formulation parameters have to be considered before reaching an optimal formulation. Based on the data obtained, scale-up using microemulsification proved to be relatively easy, mainly because it is a spontaneous process and once appropriate proportions of the constituents (surfactant, lipid and water) are obtained they will always result in a microemulsion. Furthermore, the final particle size of the formed SLNs is mainly monitored by the particle size of the microemulsion which will only form when the particle size is in the nano range.

\section{Conclusions}

In conclusion, the SLN preparation process was successfully scaled-up to a $100 x$ batch on a laboratory scale, was easy to perform and allowed reproducible SLN dispersions to be obtained. Research is ongoing for the second step of the scale-up design to produce batches of over $20 \mathrm{~L}$, and then subsequently $200 \mathrm{~L}$.

\section{References}

1. Thiraviam G, Bhandari R, Kaur IP. Sesamol: an efficient antioxidant with potential therapeutic benefits. Med Chem 2009;5:367-71.

2. Sharma S, Kaur IP. Development and evaluation of sesamol as an antiaging agent. Int J Dermatol 2006;45:200-8.

3. Kaur IP, Saini A. Sesamol exhibits antimutagenic activity against oxygen species mediated mutagenicity. Mut Res 2000;470:71-4.

4. Kapadia GJ, Azuine MA, Tokuda H, et al. Chemopreventive effect of resveratrol, sesamol, sesame oil and sunflower oil in Epstein-Barr virus early antigen activation assay and the mouse skin two-stage carcinogenesis. Pharmacol Res 2002;45:499505.

5. Vennila L, Pugalendi KV. Protective effect of sesamol against myocardial infarction caused by isoproterenol in Wistar rats. Redox Rep 2010;15:36-42.

6. Arida A, Al-Tabakha MM. Encapsulation of ketoprofen for controlled drug release. Eur J Pharm Biopharm 2007;66:48-54.

7. Kaur IP, Bhandari R, Bhandari S, Kakkar V. Potential of solid lipid nanoparticles for brain targeting. J Control Release 2008;127:97-109.

8. Shegokar R, Singh KK, Müller RH. Production and stability of stavudine solid lipid nanoparticles - From lab to industrial scale. Int J Pharm 2011;106:461-70.

9. Gasco MR. Solid lipid nanospheres from warm microemulsions. Pharm Technol Eur 1997; 9:52-8.
10. Marengo E, Cavalli R, Caputo 0, et al. Scale-up of the preparation process of solid lipid nanospheres. Part I. Int J Pharm 2000;205:3-13.

11. Kakkar V, Mishra A, Chuttani K, et al. Delivery of sesamol loaded solid lipid nanoparticles to brain for menopause related emotional and cognitive central nervous system derangements. Rejuv Res 2011;14:597-604.

12. Manjunath K, Reddy JS, Venkateswarlu V. Solid lipid nanoparticles as drug delivery systems. Methods Find Exp Clin Pharmacol 2005;27:127.

13. Orecchioni AM, Duclairior C, Depraetere P, et al. Evaluation of gliadins nanoparticles as drug delivery systems. a study of three different drugs. Int J Pharm 2003; 253:133-44.

14. Venkateswarlu V, Manjunath K. Preparation, characterization and in vitro release kinetics of clozapine solid lipid nanoparticles. J Control Release 2004;95: 627-38.

15. Üner M. Preparation, characterization and physico-chemical properties of solid lipid nanoparticles (SLN) and nanostructured lipid carriers (NLC): their benefits as colloidal drug carriers systems. Pharmzie 2006;61:375-86.

16. Müller RH, Maassen S, Weyhers H, Mehnert W. Phagocytic uptake and cytotoxicity of solid liid nanoparticles (SLN) stearically stablized with poloxamine 908 and poloxamer 407. J Drug Target 1996;4: 161-70.

17. Gasco MR. Method for producing solid lipid microspheres having a narrow size distribution. US Patent: 5250236; 1993.

18. Muchow M, Maincent P, Müller RH. Lipid nanoparticles with a solid matrix (SLN, NLC, LDC) for oral drug delivery. Drug Dev Ind Pharm 2008;34:1394-405.

19. Cavelli R, Marengo E, Rodriguez L, Gasco MR. Effects of some experimental factors on the production process of solid lipid nanoparticles. Eur J Pharm Biopharm 1996;42:110-5.

20. Blasi P, Giovagnoli S, Schoubben AL, et al. Solid lipid nanoparticles for targeted brain drug delivery. Adv Drug Deliv Rev 2007;59: 454-77. 